\title{
Vascular Smooth Muscle Cell
}

\author{
Ning Zhou, Shaunrick Stoll, Christiana Leimena and \\ Hongyu Qiu
}

Additional information is available at the end of the chapter

http://dx.doi.org/10.5772/intechopen.77249

\begin{abstract}
Vascular smooth muscle cells (VSMCs) are the stromal cells of the vascular wall and are responsible for regulating arterial tone, blood pressure, and blood supply of the tissues. VSMCs display diversity in function and phenotype depending on their location within the arterial tree (large conduit vs. small resistance vessels), their embryologic origin, and their organ-dependent microenvironment. The heterogeneity of VSMCs is regulated by multiple mechanisms including intracellular signaling and changes in the VSMC microenvironment. Genetic disorders and extrinsic stimuli-induced dysfunction in VSMCs are associated with age-related vascular pathogenesis and vascular diseases, and thus are considered as a potential therapeutic target.
\end{abstract}

Keywords: vascular smooth muscle cell, blood vessel, circulation, blood pressure, stiffness

\section{Introduction}

Vascular smooth muscle cells (VSMCs) are the main cellular components of the normal blood vessel walls, interweaving with elastic fiber layers to form the vascular media that provides structural integrity. VSMCs play an important role in the regulation of blood pressure and blood distribution to various tissues of the body through dynamic contraction and relaxation in response to vasoactive stimuli such as hormones, metabolites and neurotransmitters. Morphological and biochemical studies have revealed that two distinct phenotypes of VSMCs coexist in the vessel wall, which are the differentiated contractile and the synthetic proliferative phenotypes. These two phenotypes of VSMCs are dictated by their environmental and functional requirements and also reflect differing patterns of gene expression [1-3]. 
The contractile VSMCs are characterized by specific contractile proteins, ion channels, and cellular surface receptors that regulate the contractile process. Synthetic VSMCs, also called secretory VSMCs, are characterized by significant proliferation and migration activity, such as the production of a large amount of extracellular matrix during development, in response to the physiological changes (such as long-term exercise and pregnancy) and pathological injury (such as under the conditions of inflammation, hypertension, diabetes) [4]. It has been shown that the different phenotypes of VSMCs can reversibly switch, but a nonreversible change from the contractile to the synthetic phenotype is a prerequisite for the progression of vascular disease [4]. This chapter will summarize the current state of our knowledge on the origin and the ultrastructure of the VSMCs, and the mechanisms underlying the change in the VSMC phenotypic switch. We will also outline the current progress on the role of the VSMC dysfunction in the development of the vascular diseases and the therapeutic potential of the manipulation of the VSMC gene expression in these diseases.

\section{Origin of VSMCs}

Heterogeneity within the blood vessels is critical to cardiovascular function. In order to meet distinct physiological requirements, different regions of the vasculature exhibit different physical properties. Early studies have shown that the VSMCs of the proximal large vessels (which comprises the arch of the aorta, the common carotids, the common pulmonary trunk, and the brachiocephalic artery) are derived from the neural crest (NC) (ectomesenchymal smooth muscle) [5, 6], while VMSCs of the distal vessels (which includes the abdominal aorta and the right and left carotid arteries) are derived from the mesenchyme [7, 8]. At the region of the interface of these vessels, VSMCs are derived from mixed origins, both of the ectomesenchymal and mesenchymal.

There is abundant evidences showing that the embryonic origin of the VSMCs plays an important role in vascular biology and in the response to the stimuli response $[9,10]$. Firstly, the different embryonic origins of VSMCs reflect different gene expression patterns [11]. Secondly, differentiation of VSMCs from embryonic stem cells through NC- or mesodermlineages showed that VSMC characteristics are programmed largely based on embryonic origin [12]. These distinct embryonic origin differences also converge in the adult vessels [13]. As noted earlier, the VSMCs of the proximal aorta arise from two distinct embryonic origins: the NC and the somatic mesoderm [14, 15]. This juxtaposition of the VSMCs from different embryonic origins in the aorta contributes to the specific ability of the aorta to respond to high local pressure/force loading and various chemical stimulation as well as neuronal-hormonalregulation to meet the physiological requirement of circulation. In addition, VSMCs from different embryonic origins may also be responsible for some specific pathogenesis of vascular diseases. For example, studies have shown that when vessels that are prone to atherosclerosis are placed in a vascular region that does not typically develop atherosclerosis, they retain their predisposition to disease [16]. This evidence suggests that individual VSMC characteristics may be linked to embryonic origin. However, definitive evidence that embryonic origin dictates vascular phenotype has not been fully elucidated [17]. 
While the embryonic origins of many VSMC populations are known, the exact nature of the VSMC precursor remains elusive. It has been indicated that VSMC progenitors may arise from distinct embryonic sources including the splanchnic mesoderm [18], somatic mesoderm [15, 19], neural crest (NC) [14, 20], mesothelial [21], and other embryonic cell types [22]. In the aorta, splanchnic mesodermal cells are first recruited and differentiate into VSMCs. Before the splanchnic mesoderm cells completely encircle the dorsal aorta, the cells are displaced by the somatic mesodermal cells. Differentiation of these somatic mesodermal cells begins in the ventral anterior end of the vessel. Differentiation then proceeds around the circumference of the vessel and down the length of the aorta toward the diaphragm. Meanwhile, the cardiac NC migrates down the pharyngeal arches to invade the aortic sac. A subset of the cardiac NC participates in septation of the truncus arteriosus into the aortic arch and the pulmonary trunk [23]. The rest of the cardiac NC remains in the pharyngeal arch arteries and become the VSMCs of the aortic arch and the arteries of the head and neck [20]. The border that forms between the NC-derived VSMCs of the ascending aortic arch (aAo) and mesoderm-derived VSMCs of the descending aorta (dAo) is maintained throughout development and into adulthood [14, 20]. Once cells encircle the aorta and differentiate into VSMCs, they undergo a closely regulated process of layer formation within the media.

\section{The structure and ultrastructure of VSMCs}

Under physiological conditions, VSMCs mostly express the contractile phenotype with a spindle-like shape with a length of $50-200 \mu \mathrm{m}$, a width of $2-8 \mu \mathrm{m}$. The nucleus is located in the center surrounded by smooth endoplasmic reticulum and mitochondria. The cytoplasm is rich in thick and thin myofilaments, with every thick myofilament surrounded by 15 thin myofilaments. The thick myofilaments and thin myofilaments are aggregated into myofilament units, also known as systolic units. The intracellular thin filaments are connected by dense bodies. The adjacent dense bodies are connected by intermediate filaments to form a smooth muscle network. Thin filaments and cell membranes are connected by dense patches. Smooth muscle cells are surrounded by reticulated fibrous connective tissue, including extracellular matrix secreted by VSMCs, which interlaces individual cells into clusters to be functional units [24].

\subsection{The thick filaments}

The diameter of thick filaments is $8-16 \mathrm{~nm}$, which is a myosin dimer. The myosin superfamily is subdivided into 18 categories based on their conserved motor domain and systemic development. Type II is the constituent protein of thick filaments found in multiple subtypes of striated muscle, myocardium and smooth muscle. The smooth muscle subtype is encoded by the same gene, with selective splicing producing myosin monomer SMA and SMB [25]. The $\mathrm{SMB}$ type is more expressed in phasic contractile smooth muscle such as in the bladder and smooth muscle of the small intestine [26]. The SMA type is more abundant in tensile contractile smooth muscle. Smooth muscle myosin has a molecular weight of about $50 \mathrm{kDa}$, containing 
two heavy chains and four light chains. Each heavy chain has a carboxyl-terminal tail and an amino-terminal head, containing approximately 2000 amino acids, approximately $20 \mathrm{kDa}$. The tails of the two heavy chains are wound in the form of $\alpha$-crimping spirals to form the skeleton of the thick filaments [25]. The heads of the two heavy chains are separated, face the thin filaments, and form part of the transverse bridge. Myosin is hydrolyzed by trypsin to produce about $350 \mathrm{kDa}$ heavy meromyosin and $150 \mathrm{kDa}$ light meromyosin. Hydrolyzed myosin can be produced by proteases such as papain to generate fragment S1 and S2. The fragment S2 is a helical structure. The fragment S1 is the head of myosin and can be divided into a motor domain and lever arm. The motor domain contains an actin binding site and a nucleotide binding site [27]. The lever arm contains binding site of convert domain, myosin light chain 17 (MLC17) of $17 \mathrm{kDa}$, and myosin light chain 20 (MLC 20) of $20 \mathrm{kDa}$. The torsion zone is a site where relative rotation occurs between the motor domain and lever arm to relatively slide actin and myosin. $\mathrm{MLCl7}$ is located near the torsion zone and related to the structural stability of the lever arm. MLC20 is located near the junction of S1 and S2 [27] .

\subsection{The thin filaments}

Thin filaments, which consists of acting, are 5-8 nm in diameter and $1 \mu \mathrm{m}$ in average length. Actin is the most abundant protein in eukaryotic cells and accounts for $20 \%$ of total protein weight in muscular cells. Actin monomers, which called globular actin (G-actin), are dumbbellshaped. Microfilaments are formed by the conglomeration of actin monomers into large multimers, which are called fibrous actin (F-actin) [28]. Monomeric actin consists of 375 amino acid residues with a molecular weight of $42 \mathrm{kDa}$ which has three binding sites, one for ATP binding and two for myosin binding. Actin maintains its polymer in a dynamic, polar state by hydrolyzing ATP. Some proteins are closely related to the smooth muscle filament's function including tropomyosin, caldesmon and calponin [17, 29]. VSMCs expressed at least five tropomyosin subtypes with subtype $\alpha$ displaying the highest abundance. By regulating the binding of other proteins to actin filaments, tropomyosin affects the interaction between actin and myosin and multimerization of actin [30]. The calponin protein is a $34 \mathrm{kDa}$-sized protein that is present in smooth muscle and non-muscle tissue, and is primarily calponin-l in smooth muscle, which may reduce muscle contraction by inhibiting the myosin ATP enzyme [31].

\subsection{The skeleton protein}

The skeleton protein plays an important role in maintaining cellular shape, intracellular organelle location, intracellular vesicle trafficking, cell migration, and division [32]. Like all eukaryotic cells, VSMCs mainly contain three skeleton proteins: microfilaments, intermediate filaments and microtubules. The microfilament has a diameter of about $4 \mathrm{~nm}$ and consists of linear polymerization of actin. The filaments are connected to each other through dense bodies to form a network structure and are connected to the cell membrane through dense spots. There are four types of actin isoforms in smooth muscle: $\alpha$-smooth muscle actin, $\beta$-nonmuscular actin, $\gamma$-smooth muscle actin and $\gamma$-cytoplasmic actin, all of which are distinct gene products $[33,34]$. In VSMC, $\alpha$ - smooth muscle actin is the main subtype interacting with myosin to produce contraction. Approximately $60 \%$ of large arterial vascular actin is $\alpha$-smooth 
muscle type, $20 \%$ is $\beta$-non-muscle actin and the remainder is $\gamma$-smooth muscle and $\gamma$-cytoplasmic actin. $\Gamma$-smooth muscle actin is mainly confined to the gastrointestinal muscles. Studies have shown that $\gamma$-cytoplasmic actin is confined to the cell cortex, $\alpha$-actin serpentine longitudinal full-length cells, and $\beta$-actin borders dense plaques. The diameter of the intermediate filament is about $10 \mathrm{~nm}$, which is involved in maintaining the three-dimensional structure of the cell, maintaining the proper position of the organelle in the cytoplasm, and participating in the transfer of the membrane receptor signal to the nucleus [35]. The intermediate filaments of VSMC are in the shape of the crest, and the periphery is often accompanied by a dense body [36]. It is abundantly expressed during development and decreased with cell maturation. The intermediate filaments of differentiated vascular smooth muscle are mainly composed of vimentin and desmin [37, 38]. Vimentin, which is generally found in cells of mesenchymal origin, is the major intermediate filament types of aortic smooth muscle [37]. Skeletal proteins make the different components of the VSMCs an organic three-dimensional structure, which is a dynamic process that produces adaptive changes based on changes in cell function.

\subsection{The sarcoplasmic reticulum}

The sarcoplasmic reticulum, also known as sarcoplasmic reticulum, is a specialized smooth endoplasmic reticulum in muscle cells; a phospholipid bilayer forming a capsular network, which stores a large amount of $\mathrm{Ca}^{2+}$ [39]. At rest, the sarco/endoplasmic reticulum $\mathrm{Ca}^{2+-}$ ATPase (SERCA) transport the cytosolic $\mathrm{Ca}^{2+}$ into the sarcoplasmic reticulum through hydrolyzing ATP. The inositol (1,4,5)-triphosphate receptor (IP3R) and ryanodine receptor (RyR) channel release $\mathrm{Ca}^{2+}$ into the cytoplasm when excited and thus play an important role in the regulation of contraction and relaxation [40,41]. SERCA is a type of sarcoplasmic reticulum transmembrane $\mathrm{Ca}^{2+}$ transport ATP enzyme, which can transport two $\mathrm{Ca}^{2+}$ per one ATP hydrolyzation. Three major subtypes are known: SERCA1, SERCA2 and SERCA3. SERCA2a is presented in the myocardium, skeletal muscle and multiple smooth muscle cells. The sarcoplasmic reticulum releases calcium ions to the cytoplasm through the IP3R and RyR Ca ${ }^{2+}$ channels. IP3R is a membrane glycoprotein complex composed of inositol trisphosphateactivated $\mathrm{Ca}^{2+}$ channels. It consists of four subunits with a molecular weight of approximately $300 \mathrm{kDa}$. The ratio of IP3R and RyR in vascular smooth muscle is 3:1 to 4:1. The RyR can cluster in the sarcoplasmic reticulum near the cellular membrane, so that the local release of $\mathrm{Ca}^{2+}$ is at a high concentration [40].

\section{The VSMC phenotypes and their regulations}

\subsection{The phenotypes of VSMCs}

Although the primary function of VSMCs in the adult animal and human is contraction, VSMCs maintain considerable plasticity throughout life and can exhibit a phenotypic switch during normal development, the repair of vascular injury, and in disease states $[42,43]$. During development, VSMCs exhibit a secretory phenotype that is distinct from the spindle-shaped mature, contractile phenotype present during physiological conditions in the adult. Secretory 
VSMCs contain a large number of organelles involved in protein synthesis, whereas the main component of contractile smooth muscle cells is myofilaments.

Secretory VSMCs show high proliferation rates, apparent migration activity and strong extracellular matrix synthesis [42]. These extracellular matrixes include collagen, elastin, proteoglycan, cadherin, and integrin. At the developmental stage, VSMCs form a large number of gap junctions with endothelial cells, a process that is critical for vascular maturation [44]. In contrast, the contractile phenotype of VSMCs is very low in proliferation and the migration activity and synthesis of extracellular matrix are also low. The expression of some marker proteins is different in different phenotypic smooth muscle cells, for example, PDGF-a, intercellular adhesion molecule 1 (ICAM1), I-caldesmon, osteopontin, matrix Gla protein (MGP), collagen 1 and connexin43 decrease gradually in the process of VSMC transition from secretory to contractile type [45, 46]; whereas $\alpha 1-, \beta 1$ - and $\alpha 7$ integrins, transcriptional coactivation factors myocardin, cadherin, $\alpha$-smooth muscle actin, desmin, smooth muscle protein $22 \alpha$ (SM22 $\alpha$ ), carboxypeptidase-like protein, smooth muscle calponin, h-calmodulin binding protein, aortic preferentially expressed gene1 (APEG1) cysteine-rich protein 2 (CRP2) gradually increased during VSMC transition from secretory type to contractile type [47-49] .

Importantly, calcium signaling varies between the two phenotypes. $\mathrm{Ca}^{2+}$ signals controlled by large conductance $\mathrm{K}^{+}$channels $\mathrm{K}_{\mathrm{Ca}} 1.1$, voltage-gated L-type $\mathrm{Ca}^{2+}$ channels and RyR are associated with the transcription of differentiated contractile protein markers while signals controlled by intermediate conductance $\mathrm{Ca}^{2+}$-activated $\mathrm{K}^{+}$channels $\left(\mathrm{K}_{\mathrm{Ca}} 3.1\right)$ and TRPC channels are associated with the transcription of pro-proliferative protein markers [50]. Furthermore, the expression levels of intracellular $\mathrm{Ca}^{2+}$ release channels, $\mathrm{Ca}^{2+}$-activated proteins and pumps are also altered during VSMC phenotype switching: the synthetic VSMCs lose the RyR3 and the SERCA2a pump and reciprocally regulate isoforms of the $\mathrm{ca}^{2+} /$ calmodulin-dependent protein kinase II [50]. Changes in calcium signaling molecules as a result of phenotypic switches reflect changes in the function of the VSMCs as contractility is substituted for proliferation.

Currently, smooth muscle myosin heavy chain (SM-MHC) and smoothelin are two marker proteins that identify the contractile phenotype of smooth muscle. SM-MHC was found only in smooth muscle cells in in vivo studies and was observed only in VSMCs during embryonic development; its detection sensitivity is high in cultured vascular smooth muscle. Additionally, phenotypic switching of SMCs in response to PDGF BB in vitro, or vascular injury in vivo has been shown to be associated with a loss of activating histone modifications at gene loci encoding SMC marker genes, but retention of additional markers such as H3K4 methylation [51]. It was postulated that epigenetic mechanisms may allow for the "cell lineage memory" during the reversible phenotypic switching of VSMCs [51].

\subsection{The regulation of VSMCs phenotypes}

The mature skeletal muscle is terminally differentiated, that is, the its ability to be changed is limited. However, the mature VSMC has a strong plasticity, with significant and reversible phenotypic changes occurring when the local environment changes. The VSMCs can quickly transform from a contractile phenotype to a secretory phenotype in response to injury. 
Significantly, when the injury is repaired and the local environment returns to normal condition, VSMCs can regain the contractile phenotype. Thus, the regulatory mechanisms have to be reversible.

Although the molecular mechanisms controlling the VSMCs phenotypes switching have not been fully understood and the signaling pathways involved under different conditions may be variant, epigenetic mechanisms have been suggested as a possible explanation of the reversibility of VSMC switching. For example, microRNA 663 and micro RNA 133 have been identified as modulators of VSMC phenotypic switching [52, 53]. In addition, it has been widely-accepted that serum response factor (SRF)-mediated signaling pathways play an important role in the regulation of VSMC phenotype changes [54]. The SRF belongs to the MADS box transcription factor superfamily, through which the cis-element CArG box binding regulates the transcription of smooth muscle marker genes and is closely related to the phenotype of smooth muscle [54]. SiRNA-mediated SRF suppression affected the expression of established SRF target genes such as SMA or SM22 $\alpha$ and decreased both Factin formation and cell migration [54]. The binding state of SRF and myocardin under normal oxygen conditions promotes the transcription and contraction phenotype of smooth muscle marker genes. The transcriptional coactivator myocardin and the nuclear transcription factor Elk1 competed for the same binding site on the SRF. The binding state of SRF and myocardin in pulmonary vascular smooth muscle under normal oxygen conditions promotes the transcription and contraction phenotype of smooth muscle marker genes, and the expression of myocardin decreases under hypoxia, leading to enhanced binding of SRF to Elk1 and smooth muscle orientation [55]. Notably, the transcription of smooth muscle cell markers can also be under epigenetic control [56]. Inhibition of histone acetyl transferases (HATs) and histone deacetylases (HDACs) decreases the activity of the promoters of SMC marker genes [57].

The expression of myocardin is down-regulated during hypoxia, leading to enhanced binding of SRF. Elevated PDGF-BB can also promote Elk1 phosphorylation and Elk1-substituted myocardin binding to SRF through the ras/raf/mek/erk kinase pathway, promoting conversion to the secretory phenotype [58]. Activation of RhoA is essential for smooth muscle specific transcriptional up-regulation. RhoA activates multimerization of actin via ROCK and then promotes the translocation of myocardin-related transcription factor (MRTF) into the nucleus and binds to SRF, stimulating smooth muscle marker gene transcription and conversion to a contractile phenotype [59]. Rho-dependent MRTF nuclear translocation is one of the key regulation mechanisms of smooth muscle cell differentiation.

\section{VSMCs dysfunction in vascular pathogenesis and diseases}

\subsection{Intrinsic VSMC mechanical property and aging-induced aortic stiffness}

An increase in vascular stiffness is a common vascular pathogenesis of aging and of agingrelated cardiovascular disease and has been assumed to be caused by molecular changes of the ECM and the dysfunction of endothelial cells in elastic arteries. It was not until recent years, 
with the use of two unique techniques, atomic force microscopy (AFM) [60] and a reconstituted tissue model [60], that it was discovered that both the intrinsic mechanisms in VSMCs and the alterations in VSMC-ECM interaction contribute to the increased aortic stiffness in the old non-human primates [21]. The underlying mechanisms involve the increased expression and polymerization of $\alpha$-smooth muscle actin (a stress fiber-specific isoform of actin for VSMCs), microtubules and myosin light chain kinase (MLCK), and also the increased expression of adhesion molecule $\beta 1$-integrin and its binding to fibronectin. It was also suggested that the oscillatory behavior of VSMC elasticity and adhesion are affected differently during aging, which may link these events to changes in vascular stiffness. However, the molecular mechanisms are still not fully understood [61].

\subsection{VSMCs and hypertension}

Hypertension is one of the most common cardiovascular diseases, which eventually results in heart, renal failure or stroke. Although most of previous studies focused on the changes in the ECM and impaired endothelial control [62-64], increasing evidences indicate that VSMCs play an important role in the development of hypertension. It has been shown that arterial hypertension is accompanied by the proliferation and migration of VSMCs $[65,66]$. One of the most typical features of vascular remodeling in the course of hypertension includes thickening of the middle layer and intima and the increase of the ratio of wall thickness to lumen. These changes are mainly found in the small arteries of hypertension, mainly due to hypertrophy and proliferation of VSMCs and the migration of VSMC into the intima. In addition, recent studies confirmed that the increased intrinsic stiffness of VSMCs from hypertensive aorta contribute to the aortic stiffening and high blood pressure. The underlying mechanisms are involved in the upregulation of ROCK-SRF/myocardin and $\alpha$-smooth muscle actin signaling in the VSMCs. It is noteworthy that a heterogeneity of mechanical properties in VSMCs between the large aorta and downstream distal arteries in the hypertensive model was shown, which is accompanied with a parallel regional difference of the SRF/myocardin signaling pathway. These observations further support the concept that the different origins of VSCMs plays a role in the development of hypertension. Furthermore, a most recent study from the same group also indicate that VSMCs from hypertensive aorta are able to contribute to hypertensive vessel changes by interrupting synthesis and degradation as well as organization of ECM through the regulation of activity of lysyl oxidase (LOX) and integrin $\beta 1$. Importantly, targeting the stiffening of VSMCs effectively lowered aortic stiffness and blood pressure which revealed a promising therapeutic potential of anti-hypertension treatment in the future $[28,59]$.

\subsection{VSMCs and atherosclerosis}

Studies have been shown that VSMCs play a complex role in the formation of atherosclerosis, including increased matrix synthesis, production of multiple proteases, and changes in vascular contractility, in which the proliferation and apoptosis of VSMCs play a major role in the process of intima thickening and formation of atherosclerotic plaque [67]. Prior to the development of atherosclerosis the VSMCs maintain a stable phenotypical features and showed low 
proliferation. As atherosclerosis developed, the VSMC phenotype changes to a more proliferative nature, with reduced contraction, increased proteoglycans, but reduced expression of the typical smooth muscle markers [68-70]. The biological effects of VSMCs were discovered during the in vitro culturing of these cells and they vary with different subtypes of different species, such as the spindle-shaped smooth muscle cells (S-SMC), the epithelioid-smooth muscle cell (E-SMC) and rhomboid- smooth muscle cell (R-SMC) in animal models [71-73]. The S-SMC exhibit features of the contractile phenotype, showing high expression of $\alpha$-SMA, desmin, and SM-MHC [74]. The E-SMC and R-SMC, on the other hand, exhibit the synthetic phenotype that had significantly higher proliferation and migration proficiency. The S-SMC is more sensitive to vascular contractile factors (including endothelin 1, angiotensin II, etc.) than E-SMC. Studies on the biological behavior of different smooth muscle subtypes have led to a deeper understanding of the phenomenon of deposition of smooth muscle cells in the subendothelial cells during the course of atherosclerosis. As atherosclerotic plaque grow, apoptosis in mainly macrophages and VSMCs have been detected in the plaques [75]. It has been suggested that macrophages play a role in inducing the apoptotic process in the VSMCs which may trigger plaque rupture [76, 77]. Interestingly dying VSMCs are associated with inflammation as the apoptotic process induce release of cytokines such as IL- 1 and IL- $1 \beta$ from the dying or apoptotic VSMCs [78]. Chronic VSMC apoptosis promotes the progression of atherosclerosis.

\subsection{VSMCs and aortic aneurysm}

Aortic aneurysm (AA) is a life-threatening condition where a bulge forms in the aortic wall. There are abdominal and thoracic AA. The basic structural unit of the aortic wall is the two layers of elastic fibers clamped by the VSMCs to form a sandwich structure. This structure allows the blood vessel wall to have a good contractile strength and elasticity. The abnormality of its composition and function may lead to AA. The development of AA can begin with the degradation of ECM within the media due to a proteolytic process, which loosens the wall tension created by the ECM and VSMCs [79]. Mucoid degeneration in the aortic media can induce the disappearance of VSMCs. Loss of VSMCs inhibits the clearance of proteolytic enzymes, leading to replacement by vacuoles, proteases, apoptotic cells and modified glycosaminoglycans. This state leads to the progression to chronic dilatation and development of thoracic AA [79]. Like atherosclerosis, phenotypic switching and VSMC apoptosis also influence the development and progression of AA [80]. VSMCs express NADPH oxidases isoforms, which then regulates the proliferation migration and apoptosis of the VSMCs [81, 82]. This implicates that oxidative stress also play an influential role in the development of AA. The mutations in some cytoskeletal proteins, such as MYH11, is associated with cellular contractions, may cause AA [83]. The rigidity and function of the cytoskeletal proteins is important to the function of the VSMCs. In the blood vessel wall of patients with MYH11 mutations, the axis of the cytoskeleton/membrane integrin-extracellular matrix is disrupted, there is accumulation of proteoglycans, breakage of elastic fibers and reduction in the number of VSMCs. The typical characteristics of AA include the medial membrane degeneration, abnormal VSMC arrangement and proliferation of epithelial vasodilatation [79]. In addition to systolic function, VSMCs are also capable of trans-differentiation and secretion under mechanical and 
biochemical stimuli, such as the secretion of a variety of matrix proteins through the interaction with integrins, G protein-coupled receptors (GPCR) and disks on the cell membrane surface [84].

\section{Pharmacological targets of VSMCs}

Since VSMCs play a critical role in the cardiovascular diseases such as aortic stiffening, hypertension, atherosclerosis and aortic aneurysm, they have become a therapeutic target in the treatment of these diseases. For example, atorvastatin, one of the most effective drugs for treating cardiovascular diseases, suppresses tacrolimus-stimulated VSMC proliferation via down-regulation of $\beta$-catenin, ERK1/2, and cyclin B. Tropoelastin is shown to regulate VSMC phenotypic switch and inhibit VSMC proliferation and migration [22]. Drugs, such as paclitaxel and rapamycin which are eluted in the coronary artery stent inhibit the proliferation of VSMCs and significantly reduces the restenosis rate. In addition, new basic investigation on the VSMCs in aortic stiffness during aging and hypertension also provides new targets for the treatment of vascular diseases. For example, two anti-stiffening treatment of VSMC that act either through the inhibition of ROCK-SRF/myocardin signaling by Rock inhibitor (Y-27632), or through the inhibition of the SRF/myocardin signaling (CCG-100602) have been shown to be able to reduce the aortic stiffness and high blood pressure in hypertension. These suggest that VSMCs can be a promising target of the treatment of hypertension [28, 59]. The successful preclinical application of these VSMC-targeted interventions underscores the promising prospective of medications targeting VSMC. In fact, Plumericin inhibits proliferation of VSMCs by blocking STAT3 signaling via S-glutathionylation, highlighting the feasibility of clinical manipulation of VSMCs [85, 86].

\section{Conclusions}

As summarized in the Figure 1, VSMCs are not only the dominant components of the medial layer of blood vessels, but also important endocrine cells which secret various signaling factors promoting the arterial remodeling in the case of pathological stimuli. Multiple factors including embryonic origin, regional mechanical load, pathological stimuli and genetic mutations mediate the gene expression of VSMCs through different signaling pathways which involve the VSMC membrane receptors, calcium channels, miRNAs, DNA methylation, and histone modification. This results in the regulation of VSMC phenotypes, the expression of stiffnessrelated proteins, and ECM production. These changes subsequently affect VSMCs stiffness, migration, and proliferation, as well as ECM remodeling, thus, playing a role in normal vascular physiology and diseases. Although the mechanisms involved in vascular diseases remain largely unknown, SRF/myocardin mediated signaling pathways have been identified as a key mechanism within the developmental of vascular diseases through their regulations on the VSMC stiffness, phenotypic switching and ECM remodeling. Targeting VSMC is a 


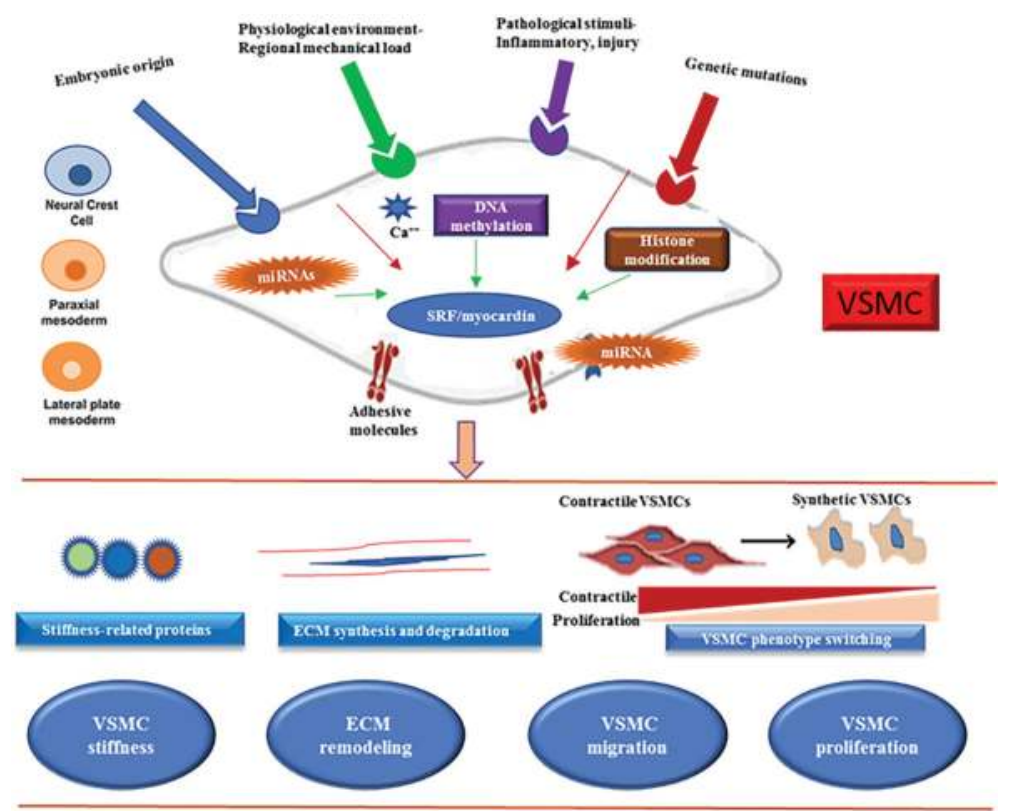

Region-Specific Vascular Physiology and Vascular Diseases

Figure 1. Summary of the role of VSMCs in normal vascular physiology and in the development of vascular diseases. Multiple factors including embryonic origin, regional mechanical load, pathological stimuli and genetic mutations mediate the gene expression of VSMCs through different signaling pathways which involves the VSMC membrane receptors, calcium channels, miRNAs, DNA methylation, and histone modification. This results in the regulation of VSMC phenotypes, the expression of stiffness-related proteins, and ECM production. These changes subsequently affect VSMCs stiffness, migration, and proliferation, as well as ECM remodeling, thus, playing a role in vascular normal physiology and diseases.

promising therapeutic of hypertension, atherosclerosis and aortic dissection/aneurisms and other related diseases.

\section{Future directions}

The pathological changes, especially the phenotypic switching of VSMC, are the most important mechanisms and characteristics of various cardiovascular diseases, including hypertension, atherosclerosis and aortic dissection/aneurisms. Medications targeting VSMCs have been clinically prescribed in the treatment of these diseases. SMC-specific drugs may be achieved through the different approaches: (1) identifying genes/proteins targets that differentially regulate VSMC phenotype changes and identifying markers of synthetic phenotype; (2) identifying genes/proteins that target intrinsic mechanical properties of the VSMCs and developing inhibitors of the proteins; (3) exploring non-coding RNAs, including microRNA, long- or short-non-coding RNA and other epigenomic alterations such as DNA methylation and 
histone regulation that differentially regulates VSMC function; (4) identifying growth factor/ hormones that have differential cellular effects on VSMC; and (5) combined usage of multiple drugs to achieve distinct functions in ECs and VSMCs.

\section{Acknowledgements}

This work is supported by the grants of 1R01 HL115195-01 and HL137962 from NIH/NHLBI (Hongyu Qiu) and the National Natural Science Fund of China (81100087, 81570261, Ning Zhou).

\section{Conflict of interest}

None.

\section{Notes/Thanks/Other declarations}

None.

\section{Author details}

Ning Zhou ${ }^{1,2}$, Shaunrick Stoll ${ }^{2}$, Christiana Leimena ${ }^{2}$ and Hongyu Qiu ${ }^{2 *}$

*Address all correspondence to: hqiu@llu.edu

1 Division of Cardiology, Department of Internal Medicine, Tongji Hospital, Tongji Medical College, Huazhong University of Science and Technology, Wuhan, China

2 Division of Physiology, Department of Basic Sciences, School of Medicine, Loma Linda University, Loma Linda, CA, USA

\section{References}

[1] Metz RP, Patterson JL, Wilson E. Vascular smooth muscle cells: Isolation, culture, and characterization. Methods in Molecular Biology. 2012;843:169-176

[2] Shanahan CM, Weissberg PL. Smooth muscle cell heterogeneity: Patterns of gene expression in vascular smooth muscle cells in vitro and in vivo. Arteriosclerosis, Thrombosis, and Vascular Biology. 1998;18:333-338

[3] Weissberg PL, Cary NR, Shanahan CM. Gene expression and vascular smooth muscle cell phenotype. Blood Pressure. Supplement. 1995;2:68-73 
[4] Lv P, Zhang F, Yin YJ, Wang YC, Gao M, Xie XL, Zhao LL, Dong LH, Lin YL, Shu YN, Zhang DD, Liu GX, Han M. SM22alpha inhibits lamellipodium formation and migration via Ras-Arp 2/3 signaling in synthetic VSMCs. American Journal of Physiology. Cell Physiology. 2016;311:C758-C767

[5] Bergwerff M, DeRuiter MC, Hall S, Poelmann RE, Gittenberger-de Groot AC. Unique vascular morphology of the fourth aortic arches: Possible implications for pathogenesis of type-B aortic arch interruption and anomalous right subclavian artery. Cardiovascular Research. 1999;44:185-196

[6] Isayama N, Matsumura G, Yamazaki K. Comparison of vascular smooth muscle cells in canine great vessels. BMC Veterinary Research. 2013;9:54

[7] Akeson AL, Greenberg JM, Cameron JE, Thompson FY, Brooks SK, Wiginton D, Whitsett JA. Temporal and spatial regulation of VEGF-A controls vascular patterning in the embryonic lung. Developmental Biology. 2003;264:443-455

[8] El-Hashash AH, Al Alam D, Turcatel G, Bellusci S, Warburton D. Eyes absent 1 (Eya1) is a critical coordinator of epithelial, mesenchymal and vascular morphogenesis in the mammalian lung. Developmental Biology. 2011;350:112-126

[9] Cheung C, Bernardo AS, Pedersen RA, Sinha S. Directed differentiation of embryonic origin-specific vascular smooth muscle subtypes from human pluripotent stem cells. Nature Protocols. 2014;9:929-938

[10] Pfaltzgraff ER, Bader DM. Heterogeneity in vascular smooth muscle cell embryonic origin in relation to adult structure, physiology, and disease. Developmental Dynamics. 2015; 244:410-416

[11] Zhang T, Zhang X, Yu W, Chen J, Li Q, Jiao Y, He P, Shen C. Effects of chemokine-like factor 1 on vascular smooth muscle cell migration and proliferation in vascular inflammation. Atherosclerosis. 2013;226:49-57

[12] Cheung C, Bernardo AS, Trotter MW, Pedersen RA, Sinha S. Generation of human vascular smooth muscle subtypes provides insight into embryological origin-dependent disease susceptibility. Nature Biotechnology. 2012;30:165-173

[13] Pfaltzgraff ER, Shelton EL, Galindo CL, Nelms BL, Hooper CW, Poole SD, Labosky PA, Bader DM, Reese J. Embryonic domains of the aorta derived from diverse origins exhibit distinct properties that converge into a common phenotype in the adult. Journal of Molecular and Cellular Cardiology. 2014;69:88-96

[14] Jiang Y, Triggle CR. Lack of involvement of endothelin-1 in angiotensin II-induced contraction of the isolated rat tail artery. British Journal of Pharmacology. 2000;131:1055-1064

[15] Wasteson P, Johansson BR, Jukkola T, Breuer S, Akyurek LM, Partanen J, Lindahl P. Developmental origin of smooth muscle cells in the descending aorta in mice. Development. 2008;135:1823-1832

[16] Haimovici H, Maier N. Experimental canine atherosclerosis in autogenous abdominal aortic grafts implanted into the jugular vein. Atherosclerosis. 1971;13:375-384 
[17] Grootaert MOJ, Moulis M, Roth L, Martinet W, Vindis C, Bennett MR, De Meyer GRY. Vascular smooth muscle cell death, autophagy and senescence in atherosclerosis. Cardiovascular Research. 2018;114:622-634

[18] Wilm B, Ipenberg A, Hastie ND, Burch JB, Bader DM. The serosal mesothelium is a major source of smooth muscle cells of the gut vasculature. Development. 2005;132:5317-5328

[19] Esner M, Meilhac SM, Relaix F, Nicolas JF, Cossu G, Buckingham ME. Smooth muscle of the dorsal aorta shares a common clonal origin with skeletal muscle of the myotome. Development. 2006;133:737-749

[20] Le Lievre CS, Le Douarin NM. Mesenchymal derivatives of the neural crest: Analysis of chimaeric quail and chick embryos. Journal of Embryology and Experimental Morphology. 1975;34:125-154

[21] Que J, Wilm B, Hasegawa H, Wang F, Bader D, Hogan BL. Mesothelium contributes to vascular smooth muscle and mesenchyme during lung development. Proceedings of the National Academy of Sciences of the United States of America. 2008;105:16626-16630

[22] Rinkevich Y, Mori T, Sahoo D, Xu PX, Bermingham Jr JR, Weissman IL. Identification and prospective isolation of a mesothelial precursor lineage giving rise to smooth muscle cells and fibroblasts for mammalian internal organs, and their vasculature. Nature Cell Biology. 2012;14:1251-1260

[23] Kirby ML, Gale TF, Stewart DE. Neural crest cells contribute to normal aorticopulmonary septation. Science. 1983;220:1059-1061

[24] Maeng J, Sheverdin V, Shin H, Ha I, Bae SS, Yang-Yen HF, Lee K. Up-regulation of Rhoa/ Rho kinase pathway by translationally controlled tumor protein in vascular smooth muscle cells. International Journal of Molecular Sciences. 2014;15:10365-10376

[25] Fisher SA. Vascular smooth muscle phenotypic diversity and function. Physiological Genomics. 2010;42A:169-187

[26] Eddinger TJ, Meer DP. Single rabbit stomach smooth muscle cell myosin heavy chain SMB expression and shortening velocity. American Journal of Physiology. Cell Physiology. 2001;280:C309-C316

[27] Carrillo-Sepulveda MA, Barreto-Chaves ML. Phenotypic modulation of cultured vascular smooth muscle cells: A functional analysis focusing on MLC and ERK1/2 phosphorylation. Molecular and Cellular Biochemistry. 2010;341:279-289

[28] Zhou N, Lee JJ, Stoll S, Ma B, Wiener R, Wang C, Costa KD, Qiu H. Inhibition of SRF/ myocardin reduces aortic stiffness by targeting vascular smooth muscle cell stiffening in hypertension. Cardiovascular Research. 2017;113:171-182

[29] Kordowska J, Huang R, Wang CL. Phosphorylation of caldesmon during smooth muscle contraction and cell migration or proliferation. Journal of Biomedical Science. 2006;13: 159-172 
[30] Gallant C, Appel S, Graceffa P, Leavis P, Lin JJ, Gunning PW, Schevzov G, Chaponnier C, DeGnore J, Lehman W, Morgan KG. Tropomyosin variants describe distinct functional subcellular domains in differentiated vascular smooth muscle cells. American Journal of Physiology. Cell Physiology. 2011;300:C1356-C1365

[31] Gao H, Steffen MC, Ramos KS. Osteopontin regulates alpha-smooth muscle actin and calponin in vascular smooth muscle cells. Cell Biology International. 2012;36:155-161

[32] Huang HC, Lai MW, Wang HR, Chung YL, Hsieh LM, Chen CC. Antiproliferative effect of esculetin on vascular smooth muscle cells: Possible roles of signal transduction pathways. European Journal of Pharmacology. 1993;237:39-44

[33] Mackenzie LS, Lymn JS, Hughes AD. Linking phospholipase C isoforms with differentiation function in human vascular smooth muscle cells. Biochimica et Biophysica Acta. 2013; 1833:3006-3012

[34] Kim HR, Gallant C, Leavis PC, Gunst SJ, Morgan KG. Cytoskeletal remodeling in differentiated vascular smooth muscle is actin isoform dependent and stimulus dependent. American Journal of Physiology. Cell Physiology. 2008;295:C768-C778

[35] Molony L, Hagen PO, Schachat FH. Intermediate filament heterogeneity in normal and hypercholesterolemic rabbit vascular smooth muscle cells. Experimental Cell Research. 1986;163:78-86

[36] Osborn M, Caselitz J, Weber K. Heterogeneity of intermediate filament expression in vascular smooth muscle: A gradient in desmin positive cells from the rat aortic arch to the level of the arteria iliaca communis. Differentiation. 1981;20:196-202

[37] Kacem K, Seylaz J, Aubineau P. Differential processes of vascular smooth muscle cell differentiation within elastic and muscular arteries of rats and rabbits: An immunofluorescence study of desmin and vimentin distribution. The Histochemical Journal. 1996;28:53-61

[38] Sakata N, Kawamura K, Fujimitsu K, Chiang YY, Takebayashi S. Immunocytochemistry of intermediate filaments in cultured arterial smooth muscle cells: Differences in desmin and vimentin expression related to cell of origin and/or plating time. Experimental and Molecular Pathology. 1990;53:126-139

[39] Poburko D, Liao CH, van Breemen C, Demaurex N. Mitochondrial regulation of sarcoplasmic reticulum $\mathrm{Ca}^{2+}$ content in vascular smooth muscle cells. Circulation Research. 2009;104:104-112

[40] Johny JP, Plank MJ, David T. Importance of altered levels of SERCA, IP3R, and RyR in vascular smooth muscle cell. Biophysical Journal. 2017;112:265-287

[41] Wu KD, Bungard D, Lytton J. Regulation of SERCA Ca ${ }^{2+}$ pump expression by cytoplasmic $\mathrm{Ca}^{2+}$ in vascular smooth muscle cells. American Journal of Physiology. Cell Physiology. 2001;280:C843-C851

[42] Yoshida T, Owens GK. Molecular determinants of vascular smooth muscle cell diversity. Circulation Research. 2005;96:280-291 
[43] Owens GK. Regulation of differentiation of vascular smooth muscle cells. Physiological Reviews. 1995;75:487-517

[44] Schwartz SM. Smooth muscle migration in vascular development and pathogenesis. Transplant Immunology. 1997;5:255-260

[45] Lee HM, Kim HJ, Won KJ, Choi WS, Lee KY, Bae YM, Park PJ, Park TK, Lee YL, Lee CK, Kim B. Contribution of soluble intercellular adhesion molecule-1 to the migration of vascular smooth muscle cells. European Journal of Pharmacology. 2008;579:260-268

[46] Jiang Q, Huang R, Cai S, Wang CL. Caldesmon regulates the motility of vascular smooth muscle cells by modulating the actin cytoskeleton stability. Journal of Biomedical Science. 2010;17:6

[47] Miano JM, Olson EN. Expression of the smooth muscle cell calponin gene marks the early cardiac and smooth muscle cell lineages during mouse embryogenesis. The Journal of Biological Chemistry. 1996;271:7095-7103

[48] Huang J, Kontos CD. Inhibition of vascular smooth muscle cell proliferation, migration, and survival by the tumor suppressor protein PTEN. Arteriosclerosis, Thrombosis, and Vascular Biology. 2002;22:745-751

[49] Uglow EB, Slater S, Sala-Newby GB, Aguilera-Garcia CM, Angelini GD, Newby AC, George SJ. Dismantling of cadherin-mediated cell-cell contacts modulates smooth muscle cell proliferation. Circulation Research. 2003;92:1314-1321

[50] House SJ, Potier M, Bisaillon J, Singer HA, Trebak M. The non-excitable smooth muscle: Calcium signaling and phenotypic switching during vascular disease. Pflügers Archiv. 2008;456:769-785

[51] Owens GK. Molecular control of vascular smooth muscle cell differentiation and phenotypic plasticity. Novartis Foundation Symposium. 2007;283:174-191 (discussion 191-173, 238-141)

[52] Torella D, Iaconetti C, Catalucci D, Ellison GM, Leone A, Waring CD, Bochicchio A, Vicinanza C, Aquila I, Curcio A, Condorelli G, Indolfi C. Micro RNA-133 controls vascular smooth muscle cell phenotypic switch in vitro and vascular remodeling in vivo. Circulation Research. 2011;109:880-893

[53] Li P, Zhu N, Yi B, Wang N, Chen M, You X, Zhao X, Solomides CC, Qin Y, Sun J. Micro RNA-663 regulates human vascular smooth muscle cell phenotypic switch and vascular neointimal formation. Circulation Research. 2013;113:1117-1127

[54] Werth D, Grassi G, Konjer N, Dapas B, Farra R, Giansante C, Kandolf R, Guarnieri G, Nordheim A, Heidenreich O. Proliferation of human primary vascular smooth muscle cells depends on serum response factor. European Journal of Cell Biology. 2010;89:216-224

[55] Zhou W, Negash S, Liu J, Raj JU. Modulation of pulmonary vascular smooth muscle cell phenotype in hypoxia: Role of cGMP-dependent protein kinase and myocardin. American Journal of Physiology. Lung Cellular and Molecular Physiology. 2009;296:L780-L789 
[56] Gomez D, Swiatlowska P, Owens GK. Epigenetic control of smooth muscle cell identity and lineage memory. Arteriosclerosis, Thrombosis, and Vascular Biology. 2015;35:2508-2516

[57] Qiu P, Li L. Histone acetylation and recruitment of serum responsive factor and CREBbinding protein onto SM22 promoter during SM22 gene expression. Circulation Research. 2002;90:858-865

[58] Hinson JS, Medlin MD, Lockman K, Taylor JM, Mack CP. Smooth muscle cell-specific transcription is regulated by nuclear localization of the myocardin-related transcription factors. American Journal of Physiology. Heart and Circulatory Physiology. 2007;292: H1170-H1180

[59] Zhou N, Lee JJ, Stoll S, Ma B, Costa KD, Qiu H. Rho kinase regulates aortic vascular smooth muscle cell stiffness via actin/SRF/myocardin in hypertension. Cellular Physiology and Biochemistry. 2017;44:701-715

[60] Qiu H, Zhu Y, Sun Z, Trzeciakowski JP, Gansner M, Depre C, Resuello RR, Natividad FF, Hunter WC, Genin GM, Elson EL, Vatner DE, Meininger GA, Vatner SF. Short communication: Vascular smooth muscle cell stiffness as a mechanism for increased aortic stiffness with aging. Circulation Research. 2010;107:615-619

[61] Zhu Y, Qiu H, Trzeciakowski JP, Sun Z, Li Z, Hong Z, Hill MA, Hunter WC, Vatner DE, Vatner SF, Meininger GA. Temporal analysis of vascular smooth muscle cell elasticity and adhesion reveals oscillation waveforms that differ with aging. Aging Cell. 2012;11:741-750

[62] Lemarie CA, Tharaux PL, Lehoux S. Extracellular matrix alterations in hypertensive vascular remodeling. Journal of Molecular and Cellular Cardiology. 2010;48:433-439

[63] Zieman SJ, Melenovsky V, Kass DA. Mechanisms, pathophysiology, and therapy of arterial stiffness. Arteriosclerosis, Thrombosis, and Vascular Biology. 2005;25:932-943

[64] Qiu H, Depre C, Ghosh K, Resuello RG, Natividad FF, Rossi F, Peppas A, Shen YT, Vatner DE, Vatner SF. Mechanism of gender-specific differences in aortic stiffness with aging in nonhuman primates. Circulation. 2007;116:669-676

[65] Hixon ML, Gualberto A. Vascular smooth muscle polyploidization - from mitotic checkpoints to hypertension. Cell Cycle. 2003;2:105-110

[66] Touyz RM. Intracellular mechanisms involved in vascular remodelling of resistance arteries in hypertension: Role of angiotensin II. Experimental Physiology. 2005;90:449-455

[67] Pauly RR, Passaniti A, Bilato C, Monticone R, Cheng L, Papadopoulos N, Gluzband YA, Smith L, Weinstein C, Lakatta EG, et al. Migration of cultured vascular smooth muscle cells through a basement membrane barrier requires type IV collagenase activity and is inhibited by cellular differentiation. Circulation Research. 1994;75:41-54

[68] Orekhov AN, Andreeva ER, Mikhailova IA, Gordon D. Cell proliferation in normal and atherosclerotic human aorta: Proliferative splash in lipid-rich lesions. Atherosclerosis. 1998;139:41-48 
[69] Kolodgie FD, Burke AP, Farb A, Weber DK, Kutys R, Wight TN, Virmani R. Differential accumulation of proteoglycans and hyaluronan in culprit lesions. Insights Into Plaque Erosion. 2002;22:1642-1648

[70] Allahverdian S, Chaabane C, Boukais K, Francis GA, Bochaton-Piallat M-L. Smooth muscle cell fate and plasticity in atherosclerosis. Cardiovascular Research. 2018;114:540-550

[71] Orlandi A, Ehrlich HP, Ropraz P, Spagnoli LG, Gabbiani G. Rat aortic smooth muscle cells isolated from different layers and at different times after endothelial denudation show distinct biological features in vitro. Arteriosclerosis and Thrombosis: A Journal of Vascular Biology. 1994;14:982-989

[72] Bochaton-Piallat ML, Ropraz P, Gabbiani F, Gabbiani G. Phenotypic heterogeneity of rat arterial smooth muscle cell clones. Implications for the Development of Experimental Intimal Thickening, Arteriosclerosis, Thrombosis, and Vascular Biology. 1996;16:815-820

[73] Hao H, Ropraz P, Verin V, Camenzind E, Geinoz A, Pepper MS, Gabbiani G, BochatonPiallat ML. Heterogeneity of smooth muscle cell populations cultured from pig coronary artery. Arteriosclerosis, Thrombosis, and Vascular Biology. 2002;22:1093-1099

[74] Frid MG, Moiseeva EP, Stenmark KR. Multiple phenotypically distinct smooth muscle cell populations exist in the adult and developing bovine pulmonary arterial media in vivo. Circulation Research. 1994;75:669-681

[75] Bennett MR, Evan GI, Schwartz SM. Apoptosis of human vascular smooth muscle cells derived from normal vessels and coronary atherosclerotic plaques. The Journal of Clinical Investigation. 1995;95:2266-2274

[76] Boyle JJ, Weissberg PL, Bennett MR. Human macrophage-induced vascular smooth muscle cell apoptosis requires NO enhancement of Fas/Fas-L interactions. Arteriosclerosis, Thrombosis, and Vascular Biology. 2002;22:1624-1630

[77] Bennett MR. Apoptosis of vascular smooth muscle cells in vascular remodelling and atherosclerotic plaque rupture. Cardiovascular Research. 1999;41:361-368

[78] Clarke MCH, Talib S, Figg NL, Bennett MR. Vascular smooth muscle cell apoptosis induces Interleukin-1-directed inflammation. Effects of Hyperlipidemia-Mediated Inhibition of Phagocytosis. 2010;106:363-372

[79] Michel J-B, Jondeau G, Milewicz DM. From genetics to response to injury: Vascular smooth muscle cells in aneurysms and dissections of the ascending aorta. Cardiovascular Research. 2018;114:578-589

[80] Mao N, Gu T, Shi E, Zhang G, Yu L, Wang C. Phenotypic switching of vascular smooth muscle cells in animal model of rat thoracic aortic aneurysm. Interactive Cardiovascular and Thoracic Surgery. 2015;21:62-70

[81] Parastatidis I, Weiss D, Joseph G, Taylor WR. Overexpression of catalase in vascular smooth muscle cells prevents the formation of abdominal aortic aneurysms. Arteriosclerosis, Thrombosis, and Vascular Biology. 2013;33:2389-2396 
[82] Clempus RE, Griendling KK. Reactive oxygen species signaling in vascular smooth muscle cells. Cardiovascular Research. 2006;71:216-225

[83] Takeda N, Morita H, Fujita D, Inuzuka R, Taniguchi Y, Nawata K, Komuro I. A deleterious MYH11 mutation causing familial thoracic aortic dissection. Human Genome Variation. 2015;2:15028

[84] Lacolley P, Regnault V, Segers P, Laurent S. Vascular smooth muscle cells and arterial stiffening: Relevance in development, aging, and disease. Physiological Reviews. 2017;97: $1555-1617$

[85] Heiss EH, Liu R, Waltenberger B, Khan S, Schachner D, Kollmann P, Zimmermann K, Cabaravdic M, Uhrin P, Stuppner H, Breuss JM, Atanasov AG, Dirsch VM. Plumericin inhibits proliferation of vascular smooth muscle cells by blocking STAT3 signaling via Sglutathionylation. Scientific Reports. 2016;6:20771

[86] Huang CH, Ciou JS, Chen ST, Kok VC, Chung Y, Tsai JJ, Kurubanjerdjit N, Huang CF, Ng KL. Identify potential drugs for cardiovascular diseases caused by stress-induced genes in vascular smooth muscle cells. Peer Journal. 2016;4:e2478 
